\title{
BMJ Open Walking-related digital mobility outcomes as clinical trial endpoint measures: protocol for a scoping review
}

\begin{abstract}
Ashley Marie Polhemus (D) , ${ }^{1}$ Ronny Bergquist, ${ }^{2}$ Magda Bosch de Basea, ${ }^{3,4}$ Gavin Brittain, ${ }^{5,6}$ Sara Catherine Buttery (D) ,7 Nikolaos Chynkiamis, ${ }^{8}$ Gloria dalla Costa, ${ }^{9}$ Laura Delgado Ortiz, ${ }^{3,4}$ Heleen Demeyer, ${ }^{10,11}$ Kirsten Emmert, ${ }^{12}$ Judith Garcia Aymerich, ${ }^{3,4}$ Heiko Gassner, ${ }^{13}$ Clint Hansen, ${ }^{12}$ Nicholas Hopkinson, ${ }^{7}$ Jochen Klucken, ${ }^{13}$ Felix Kluge, ${ }^{14}$ Sarah Koch, ${ }^{3,4}$ Letizia Leocani, ${ }^{9}$ Walter Maetzler, ${ }^{12}$ M Encarna Micó-Amigo, ${ }^{15}$ A Stefanie Mikolaizak, ${ }^{16}$ Paolo Piraino, ${ }^{17}$ Francesca Salis, ${ }^{18}$ Christian Schlenstedt (D) , ${ }^{12}$ Lars Schwickert, ${ }^{16}$ Kirsty Scott, ${ }^{19,20}$ Basil Sharrack, ${ }^{5,6}$ Kristin Taraldsen, ${ }^{2}$ Thierry Troosters, ${ }^{21}$ Beatrix Vereijken, ${ }^{2}$ loannis Vogiatzis, ${ }^{8}$ Alison Yarnall, ${ }^{15}$ Claudia Mazza, ${ }^{19,20}$ Clemens Becker, ${ }^{16}$ Lynn Rochester, ${ }^{15}$ Milo Alan Puhan, ${ }^{1}$ Anja Frei $^{1}$
\end{abstract}

To cite: Polhemus AM, Bergquist R, Bosch de Basea M, et al. Walking-related digital mobility outcomes as clinical trial endpoint measures: protoco for a scoping review. BMJ Open 2020;10:e038704. doi:10.1136/ bmjopen-2020-038704

- Prepublication history and additional material for this paper are available online. To view these files, please visit the journal online (http://dx.doi org/10.1136/bmjopen-2020038704).

Received 20 March 2020 Revised 14 May 2020 Accepted 18 May 2020

Check for updates

(c) Author(s) (or their employer(s)) 2020. Re-use permitted under CC BY-NC. No commercial re-use. See rights and permissions. Published by BMJ.

For numbered affiliations see end of article.

Correspondence to Ashley Marie Polhemus; ashley.polhemus@uzh.ch

\section{ABSTRACT}

Introduction Advances in wearable sensor technology now enable frequent, objective monitoring of real-world walking. Walking-related digital mobility outcomes (DMOs), such as real-world walking speed, have the potential to be more sensitive to mobility changes than traditional clinical assessments. However, it is not yet clear which DMOs are most suitable for formal validation. In this review, we will explore the evidence on discriminant ability, construct validity, prognostic value and responsiveness of walkingrelated DMOs in four disease areas: Parkinson's disease, multiple sclerosis, chronic obstructive pulmonary disease and proximal femoral fracture.

Methods and analysis Arksey and O'Malley's methodological framework for scoping reviews will guide study conduct. We will search seven databases (Medline, CINAHL, Scopus, Web of Science, EMBASE, IEEE Digital Library and Cochrane Library) and grey literature for studies which (1) measure differences in DMOs between healthy and pathological walking, (2) assess relationships between DM0s and traditional clinical measures, (3) assess the prognostic value of DMOs and (4) use DMOs as endpoints in interventional clinical trials. Two reviewers will screen each abstract and full-text manuscript according to predefined eligibility criteria. We will then chart extracted data, map the literature, perform a narrative synthesis and identify gaps.

Ethics and dissemination As this review is limited to publicly available materials, it does not require ethical approval. This work is part of Mobilise-D, an Innovative Medicines Initiative Joint Undertaking which aims to deliver, validate and obtain regulatory approval for DMOs. Results will be shared with the scientific community and general public in cooperation with the Mobilise-D communication team.

Registration Study materials and updates will be made available through the Center for Open Science's OSFRegistry (https://osf.io/k7395).

\section{Strengths and limitations of this study}

This is the first scoping review to explore existing evidence on the discriminant ability, construct validity, prognostic value and responsiveness of walkingrelated digital mobility outcomes.

- A broad review strategy enables identification of trends across methods and settings in four chronic conditions.

- A multidisciplinary team of clinicians, technologists, movement specialists and epidemiologists from academia and industry will conduct this review.

- Terminology and methodology associated with gait assessments are diverse and fragmented, posing limitations for study identification and synthesis.

- Following scoping review guidelines, neither critical appraisal nor meta-analysis will be conducted, limiting our ability to assess the strength of existing evidence.

\section{BACKGROUND}

For people living with chronic health conditions, walking impairment is associated with reduced quality of life, ${ }^{1-4}$ disability progression, ${ }^{156}$ fall risk, ${ }^{7-9}$ hospitalisation ${ }^{10}{ }^{11}$ and mortality. ${ }^{10}{ }^{11}$ Research is booming on therapies that can mitigate the high human and economic costs of walking impairment. However, before these therapies can be adopted in clinical practice, their efficacy must be established through controlled clinical trials. The endpoint measures used to assess these interventions' efficacy should be valid, sensitive, easy to administer and representative of real-world function or behaviour. $^{12}$ 
Unfortunately, current mobility measures pose critical limitations. Clinical trials traditionally employ two types of mobility assessments: patient-reported outcome (PROs) instruments and clinical gait assessments. PROs enable patients to report perceptions of their own mobility in a standardised manner, ${ }^{13}$ though results may be subject to recall bias. ${ }^{14-16}$ Clinical assessments, such as timed walking tests, are typically more objective. However, many still require clinical interpretation and are subject to high inter-rater variability. ${ }^{17} 18$ For example, Zhang et al conducted a sensitivity analysis to demonstrate the potential impact of inter-rater variability in clinical trials by assessing a trial's primary outcome, the Expanded Disability Status Score (a common measure of function and ambulation in multiple sclerosis) in duplicate. ${ }^{19}$ Duplicated ratings differed in over $30 \%$ of patients, affecting estimates of treatment effect. Additionally, clinical assessments are often infrequently acquired and may not be representative of real-world behaviour. ${ }^{142021}$ Compared with real-world walking, patients consistently walk faster and produce higher quality gait patterns during 'normal' walking in laboratory settings. ${ }^{20} 2223$ These challenges have prompted calls for more sensitive, reliable mobility measures in clinical trials. ${ }^{21} 24$

Advances in wearable sensor technology now enable frequent, objective mobility monitoring. Digital mobility outcomes (DMOs) such as gait speed, variability and symmetry have been used to quantify real-world walking, ${ }^{25-29}$ and emerging evidence suggests that they may be more sensitive to subtle changes than traditional instruments. ${ }^{14} 2130-32$ While a growing body of evidence supports this theory, ${ }^{12} 313334$ the validity of DMOs is not well established. ${ }^{121430}$ The field's fragmentation by disease area, technology, taxonomy and methodology 14 27 35-39 currently limits our understanding of their potential. To date, no overarching view of the clinical utility of DMOs exists. ${ }^{14}$ Thus, this study will map existing evidence on walking-related DMOs to assess their suitability for formal validation.

\section{STUDY RATIONALE AND OBJECTIVES}

This work is part of Mobilise-D, a research programme sponsored by the European Union's Innovative Medicines Initiative Joint Undertaking, which aims to deliver, validate and obtain regulatory approval for a suite of real-world DMOs. ${ }^{40}{ }^{41}$ This study will hone our understanding of the contexts and purposes for which DMOs might be most effectively used as research instruments. Our primary objective is to map the evidence describing the discriminant ability, construct validity, prognostic value and responsiveness of walking-related DMOs. We will focus on four disease areas: Parkinson's disease (PD), multiple sclerosis (MS), chronic obstructive pulmonary disease (COPD) and proximal femoral fracture (PFF). By mapping the literature and providing a narrative synthesis of our findings, we will identify which DMOs pose the greatest potential as clinical endpoints.
Box 1 Review objective and research questions (RQs)

Objective: Map existing evidence describing the discriminant ability, construct validity, prognostic value and responsiveness of walkingrelated digital mobility outcomes (DMOs)

- RQ1: What differences in DM0s have been identified between the four included populations and healthy controls?

- RQ2: What is the evidence on the associations between DMOs and clinically relevant measures of physical function, health-related quality of life, symptoms and disease severity in each of the included populations?

- RQ3: What is the evidence on the prognostic value of DMOs in each of the included populations?

- RQ4: In which contexts and for what purposes have DMOs been used as endpoints in controlled interventional studies in each of the included populations?

\section{METHODS AND ANALYSIS \\ Protocol structure}

This study employs the scoping review methodology developed by Arksey and O'Malley ${ }^{42}$ and advanced by Levac et al. ${ }^{43}$ Arksey and O'Malley's framework describes six stages of scoping review conduct: (1) identifying the research question $(\mathrm{RQ})$, (2) identifying relevant studies, (3) selecting studies, (4) charting the data, (5) collating, summarising and reporting results and (6) consulting with relevant stakeholders. In contrast to systematic reviews, which assess the literature to answer narrow RQs, scoping reviews explore a research topic from a broader perspective. They aim to map the state of evidence in a structured yet reflexive manner to identify research gaps or assess the feasibility of future systematic reviews. ${ }^{42-44}$

Here we present a harmonised review strategy stratified by RQ and population. This approach will allow us to explore the nuances of DMO research in individual disease areas while identifying overarching trends.

\section{Stage 1: identifying the $\mathbf{R Q}$}

Research questions

To be used as clinical trial endpoints, measures must be valid, clinically meaningful and responsive to change. Preliminary searches revealed a highly fragmented body of literature, with no overarching review describing these characteristics. Therefore, this study will map the literature across four RQs (box 1) in a set of walking-related DMOs (box 2). Though DMOs have potential to be used in many disease areas, this study will focus on PD, MS, COPD and PFF (subsequently referred to as included populations'). The Mobilise-D consortium selected these disease areas as exemplars for DMO development due to their diverse aetiologies of mobility impairment, high public health burden and existing evidence base. ${ }^{26274546}$

\section{RQ1: discriminant ability}

First, we will explore DMOs' discriminant ability by identifying studies which compare healthy and pathological gait (box 1, RQ1). In this analysis, we will map evidence describing differences in DMOs between people with 
Box 2 Walking-related digital mobility outcomes included in this review*

\section{Spatial parameters \\ - Step length (magnitude, variability, symmetry). \\ - Stride length (magnitude, variability). \\ - Step width (magnitude, variability). \\ Temporal parameters \\ - Cadence (magnitude, variability). \\ - Step time (magnitude, variability, symmetry). \\ - Stride time (magnitude, variability). \\ - Stance time (magnitude, variability, symmetry). \\ - Swing time (magnitude, variability, symmetry). \\ - Single support time (magnitude, variability, symmetry). \\ - Double support time (magnitude, variability). \\ Spatiotemporal parameters \\ - Gait speed (magnitude, variability). \\ - Stride speed (magnitude, variability). \\ Volume of walking \\ - Walking time. \\ - Step count. \\ - Number/duration of walking bouts. \\ ${ }^{*}$ A narrative definition of each parameter is provided in online supplementary file 1}

one of the four target diseases and healthy controls. We hypothesise that differences in some, but not all, DMOs will emerge between healthy individuals and the four included populations.

\section{RQ2: construct validity}

We will then gather evidence informing the construct validity of DMOs. We hypothesise that DMOs will exhibit moderate to strong associations with measures that assess physical function, such as balance tests, and weaker associations with measures which are not directly related to physical function. To test this, we will map cross-sectional relationships (ie, assessed across a study population at a single timepoint) between DMOs and clinically relevant measures of disease severity, physical function, healthrelated quality of life and other symptoms in each of the included populations.

\section{RQ3: prognostic value}

Next, we will map the evidence that informs the prognostic value of DMOs (ie, their ability to predict future health outcomes). We will do this by mapping longitudinal associations between DMOs measured at baseline and clinically relevant health outcomes assessed at follow-up. We hypothesise that DMOs will exhibit prognostic value similar to that established for traditional measures of mobility. ${ }^{1047-49}$

\section{RQ4: responsiveness to intervention}

Finally, we will gather evidence that informs the responsiveness of DMOs to intervention. We expect that the use of DMOs as endpoints in interventional studies will be rare. ${ }^{34}$ However, we hypothesise that, when they are used, DMOs will be responsive to interventions which improve physical function or reduce mobility-limiting symptoms. To this end, we will map the use and responsiveness of DMOs as endpoints in controlled interventional studies.

\section{Definitions and study scope}

Preliminary searches revealed that an exhaustive review is infeasible due to inconsistent terminology and reporting practices. Thus, we do not necessarily intend to produce an exhaustive list of all previous studies. Instead, we will adopt a semistructured approach to map clinically relevant trends across this large, fragmented body of literature. To do this, we will limit some dimensions of study scope to lengthy lists (ie, the DMOs, the measures assessed in RQ2 and the outcomes assessed in RQ3) and will apply basic quality thresholds (ie, a minimum number of participants). This approach allows us to remain inclusive with regard to terminology and methodology while ensuring feasibility. The decisions used to set this scope are described below. Because understanding of seemingly common terms differs across disciplines, defining the concepts addressed by this review was not trivial. Therefore, our operational definitions of key concepts such as 'mobility', 'walking', 'real-world' and 'DMOs' are clearly defined in box 3 .

\section{Mobility and real-world walking}

According to the International Classification of Functioning, Disability and Health (ICF), 'mobility' is a complex concept, inclusive of both functional ability and social participation. 'Walking' represents a distinct construct encompassed by this broader concept of mobility. In this review, we adhere to the definition of 'walking' adopted by the Mobilise-D consortium (box 3). Our ultimate aim is to explore the utility of DMOs to characterise 'real-world' walking. However, until recently, studies on gait parameters were largely confined to clinical settings. While methodologically different, laboratory or clinic-based measurements may still provide insight into DMOs' potential as real-world measures. Therefore, we will include walking-related DMOs measured in any setting, real-world or otherwise. This inclusive approach will also enable us to compare DMOs measured during real-world walking, supervised tests and scripted walking.

\section{Digital mobility outcomes}

Theoretically, DMOs could include any digital measures encompassed by the ICF definition of 'mobility.' However, our scope will be limited to a set of 32 DMOs associated with walking, (box 2) since walking is the primary focus of the Mobilise-D project. This list was compiled in consultation with mobility experts, technologists and clinicians in the four disease areas. It includes spatiotemporal parameters characterised in three widely accepted factor analyses of gait ${ }^{5-53}$ and parameters associated with daily volume of walking. This list excludes non-linear gait and dynamic balance measures, such as Lyapunov exponents ${ }^{545}$ and 
Box 3 Operational definitions of key concepts adopted for this review

\section{Mobility}

According to the International Classification of Functioning, Disability and Health (ICF), 'mobility' is defined as moving by (a) changing body position or location or by transferring from one place to another, (b) by carrying, moving or manipulating objects, (c) by walking, running or climbing and (d) by using various forms of transportation. ${ }^{65}$

\section{Walking}

Per the Mobilise-D consortium, Human walking is a method of locomotion and is defined as initiating and maintaining a forward displacement of the centre of mass in an intended direction involving the use of the two legs which provide both support and propulsion. The feet are repetitively and reciprocally lifted and set down whereby at least one foot is in contact with the ground at all times. ${ }^{667}$ Walking with walking aids is included in this definition. Walking is made up of walking bouts and is equivalent to taking steps/stepping forward (thus stepping in place does not constitute walking) and is defined as starting from initial contact for the initial step until ending with full floor contact of the foot making the last step. ${ }^{68}$

\section{Real-world walking}

Per the Mobilise-D consortium, 'Real world' relates to the context in which walking takes place- - that is free-living, unsupervised, uncontrolled and non-standardised. As such, it is unscripted as there are no instructions to the subject. Real-world actions occur in non-simulated everyday situations in unconstrained environments with minimal consciousness of being tested. It is equivalent to actions at home or in the community over continuous periods of time. ${ }^{23} \ldots$ Real-world walking is distinct from laboratory-based, ${ }^{69}$ supervised (fully controlled and observed) and semi-controlled (walking 'freely' but with supervision) tests. It also is different from scripted or instructed walking, which can take place in the home or lab.

\section{Digital mobility outcomes}

Digital mobility outcomes are digitally measured mobility parameters used to assess an individual's health status, such as spatiotemporal gait parameters, walking bout characteristics and physical activity. In this case, 'digital' measures refer to those objectively derived from electronic systems, as opposed to qualitative, paper-based or self-reported measures.

\section{Clinically relevant measures and outcomes}

'Clinically relevant' measures and outcomes as those that are routinely and broadly used either in clinical practice or in major pharmaceutical or epidemiological studies. 'Measures' refer to instruments or tests that assess an aspect of a patient's health at a single point in time, while 'outcomes' refer to identified changes in health status that result from the handling of a health problem. ${ }^{70}$

detrended fluctuation analyses, ${ }^{56}$ due to the emergent nature of their evidence base. Though we also consider digital measures of physical activity to be DMOs, physical activity measures indirectly related to walking, such as daily energy expenditure or activity intensity, are also out of scope. This is because physical activity represents a related, yet broader construct. ${ }^{57-59}$

\section{Clinically relevant measures and outcomes}

To ensure study scope remains clear and manageable, lists of included measures (46 general, 67 disease-specific) and outcomes (13 general, 16 disease-specific) were defined a-priori in consultation with technical and clinical subject matter experts on the Mobilise-D research team. While these lists are not exhaustive, they contain the most important measures and outcomes used clinically in each of the four populations (online supplementary file 1). In alignment with the reflexive approach outlined by Arksey and O'Malley, ${ }^{42}$ we defined a systematic method to amend these lists if additional instruments meeting these criteria are identified during study conduct.

\section{Stage 2: identifying relevant studies}

This study will be conducted between November 2019 and December 2020. We will include peer-reviewed and grey literature, including journal articles, reports, research letters, conference papers, doctoral theses and other publications reporting original results. MEDLINE, CINAHL, Scopus, Web of Science, EMBASE, IEEE Digital Library and the Cochrane Library will be searched for eligible peerreviewed literature. ACM Digital Library, ProQuest Dissertations, Open Grey and the National Information Center's Health Services Research Projects in Progress Database will be searched to identify relevant grey literature. We will supplement these searches with the first 100 results on Google Scholar for each population, with results first sorted by relevance and then by time. Though we will limit studies based on availability of English-language abstracts, we will include full-text articles written in any language spoken by members of the Mobilise-D consortium. Additional sources will include manual searches of reference lists and publications from the review team's private libraries. Based on subject matter expert recommendations, the search will be limited to studies published during or after 1999. This time frame reflects advances in gait monitoring technology in the early 2000s and is supported by the findings of previous systematic reviews. ${ }^{60-62}$

Due to the diverse terminology associated with digital technologies and gait assessment, we opted for a broad search strategy which was agnostic to methodology or technology. This strategy was developed through collaboration between the research team and an experienced information specialist. Each search includes terms related to walking assessments and the four populations according to the structure (walking terms) AND (population terms). The proposed search strategy for EMBASE is provided in online supplementary file 2 .

Study design, review conduct, records of deduplication, reference exclusion and individual author contributions will be managed in DistillerSR (Evidence Partners, Ottawa, Canada). Initially, references will be compiled in Endnote (Clarivate Analytics, Boston, USA), and the final review libraries will be compiled in Mendeley (Elsevier B.V., Amsterdam, The Netherlands), an open-access reference management software.

\section{Stage 3: study selection}

Study selection process

Study selection will include three steps: piloting, title and abstract screening and full-text review. All reviewers 
will receive training on scoping review conduct prior to abstract screening. All reviewers will pilot eligibility criteria on a random set of 50 abstracts to ensure consistency. Clarifications will be made as necessary. Agreement with the lead reviewer (AMP) will then be monitored on an additional 100 abstracts per reviewer at the onset of the screening process. In the full-text review stage, a similar process will be repeated on 15 full-text articles. Prior to screening, duplicate studies will be identified by comparing titles, authors, publication years and abstracts. If additional duplicates are identified during full-text review or data extraction, they will be excluded. Multiple sources reporting on the same study will be linked and analysed as one study during synthesis.

Up to three reviewers will independently screen each abstract for inclusion according to predefined eligibility criteria. Reviewers will use detailed reference sheets to ensure a uniform approach to screening (online supplementary file 3). Abstracts will proceed to full-text review if any single reviewer determines that it meets eligibility criteria. If the first reviewer includes the abstract, it will proceed automatically to full-text review and will not undergo a second screening. Agreement of two reviewers will be required to exclude an abstract. In cases of high uncertainty, reviewers will be able to request a third screening by the lead reviewer (AMP). Agreement will be assessed between each reviewer and the primary reviewer via Cohen's Kappa and as a group via Fleiss' Kappa. In contrast to Cohen's Kappa, which calculates agreement of two independent raters, Fleiss' Kappa statistic assesses reliability between any number of raters giving categorical ratings to a fixed number of items. ${ }^{63}$ We anticipate that disease-specific knowledge will be necessary for full-text eligibility assessment. Thus, studies will be manually segmented by disease area during abstract screening. Consistent with the reflexive nature of scoping reviews, a second round of abstract screening may be conducted if additional eligibility criteria are identified during the study process or if disease-specific knowledge is required to assess abstract eligibility. This round will follow the same procedure as the original screening stage.

Two reviewers will then independently assess each fulltext article for inclusion according to predefined eligibility criteria. Reason(s) for exclusion will be documented and agreement will be calculated. Reviewers will resolve disagreements through discussion. If no consensus can be reached, a senior team member will review the article and make the final determination.

\section{Eligibility criteria}

To be included, studies must address one of our RQs with respect to an included DMO in one of our four populations. Detailed criteria and considerations regarding study design, included DMOs and patient populations are provided below. We also provide operational definitions of 'addressing a RQ'.

\section{Study design and setting}

Studies must present original data to be eligible for inclusion. To prevent crowding of results, we will also require that a minimum of 10 individuals per study arm are included in a relevant analysis. Though these two criteria naturally exclude reviews, case studies and case series, any other design is theoretically eligible. However, not all study designs are capable of addressing all four RQs. Therefore, RQ-specific study design considerations are also provided as appropriate below. We will include studies and walking assessments conducted in any setting (laboratory, in-clinic, real-world).

\section{DMOs, technologies and methods}

For reasons described above, this review will be limited to the DMOs summarised in box 2. We will include DMOs produced through any digital or electronic measurement method, including wearable sensors, instrumented walkways or treadmills, optometric systems, force plates, mobile phones, stopwatches and pedometers, among others. We will include DMOs measured during any test or walking condition that includes or simulates normal, over-ground walking. This includes walking at any speed (ie, top-speed, self-selected), any start conditions (eg, static start or rolling start), single-task or dual-task walking, straight or curvilinear walking. Walking may be free or scripted, measured indoors or outdoors, supervised or unsupervised, on a treadmill, walkway or over-ground on any course regardless of shape or length. Because traditional timed gait speed tests use stopwatches (an included technology) to measure gait speed (an included DMO), they will also be included. However, we will exclude testing conditions that purposefully alter participants' normal gait patterns, such as stepping in time to music. We will also exclude analyses limited to climbing, turning and analyses of single steps, such as the recovery step after a push.

During pilots, these criteria were consistently interpreted in most cases. However, specific challenges arose regarding the eligibility of timed clinical assessments that include periods of walking. For example, some authors refer to the timed up and go (TUG) test as a measure of gait speed. However, the TUG measures the time required to rise from a seated position, walk around a course and return to a seated position. ${ }^{64}$ In populations with mobility impairment, time spent standing and sitting may not be trivial. Thus, the TUG encompasses multiple constructs. To ensure consistency, it was necessary to define explicit eligibility criteria for common tests such as the TUG. These criteria were developed based on literature searches and in consultation with mobility experts (authors LR, JGA, TT, MP, AY, LL and BS), and are described with rationale in online supplementary file 1. Generally, we will include timed gait speed tests, such as the 10-m walk, but will exclude timed tests that aggregate or assess constructs other than gait speed. However, studies may still be included if DMOs were specifically assessed during the walking portions of excluded tests. 
For example, if the TUG was instrumented and gait speed or other DMOs were measured during the walking portion of the test, this analysis is included though the total time to complete the TUG is not.

\section{Patient populations}

To be eligible, studies must include patients with a confirmed diagnosis of one of the four included conditions: PD, MS, COPD and PFF. In this context, a 'confirmed diagnosis' is one made by a clinician based on the relevant diagnostic criteria at the time of the study's publication. Further detail is provided in online supplementary file 1 . No eligibility criteria on age range, disease severity level or subtype will be applied except for PFF populations, which will be limited to adults 65 years of age or older. Studies with mixed populations will also be included if a subanalysis is conducted on an included population.

\section{Addressing an $R Q$}

Though we do not pose specific methodological requirements for inclusion, not all statistical methods and study designs are capable of addressing each RQ. For example, case-control designs cannot assess responsiveness to intervention (RQ4), though they can be used to compare pathological and healthy gait (RQ1) and assess relationships between DMOs and clinically relevant measures (RQ2). In light of these methodological distinctions, each of our RQs could be viewed as separate-though highly inter-related-reviews, harmonised under a common strategy. With this in mind, we will map the literature separately for each $\mathrm{RQ}$ and must therefore set specific criteria to determine whether studies address an RQ.

Studies will be eligible to address RQ1 if they compare an included DMO between healthy controls and one of the included populations. No other RQ-specific eligibility criteria will be applied.

Studies will be eligible to address RQ2 if they assess the relationship between an included DMO and an included measure in one of the four populations at a single timepoint (ie, a cross-sectional analysis). The list of included measures, defined a-priori, is comprised of widely used measures of disease severity, health-related quality of life, physical function, cognition, mental health and other factors (online supplementary file 1). We will include any type of statistical or qualitative analysis and set no specific study design requirements, since such an analysis could be conducted within any study design.

Studies will be eligible to address RQ3 if they assess a relationship between an included DMO measured at baseline and an included outcome assessed at follow-up (ie, a longitudinal analysis). Included outcomes are described in online supplementary file 1 . Studies must be longitudinal to address this $R Q$, though we set no further criteria on the basis of methodology or study duration.

Studies will be eligible to address RQ4 if they use an included DMO as an endpoint in a controlled interventional study in an included population. Published protocols of controlled trials will be indexed for future analysis. Studies will not be excluded on the basis of intervention type, duration or follow-up frequency. We will exclude uncontrolled studies from this RQ, since they are particularly susceptible to placebo effect and other biases. However, uncontrolled interventional studies may still be included in the review if they conduct an analysis which addresses any of the other RQs. We pose no other methodological criteria for RQ4.

At the abstract stage, studies will be included in full-text review if they could possibly have conducted an included analysis, since relevant analyses are not consistently reported at the abstract level. Analyses addressing each $\mathrm{RQ}$ will be identified during full-text screening.

\section{Addressing unforeseen eligibility criteria}

Arksey and O'Malley's framework specifically allows for flexibility in the review process, ${ }^{42}$ as appropriate scope and eligibility criteria may not be initially clear when reviewing a previously unmapped research area. As research on gait is rapidly evolving, it may be necessary to adjust our eligibility criteria and lists of included walking conditions, measures and outcomes. If initial findings warrant adjustments, a proposal will be submitted to a team of project leads who will make the final determination on how to adjust eligibility criteria. Adjustments will be applied to all identified studies and reported accordingly.

\section{Stage 4: charting the data}

\section{Data extraction}

Data collection forms will be developed through iterative review with the research team and further refined through expert feedback. Forms will capture all relevant study data and contextual information while ensuring adequate flexibility to capture emerging themes. Prior to initiating data extraction, the form will be tested by reviewers on a random sample of at least five studies. Additional modifications to the form identified through this pilot will be reviewed and approved by the research team.

Data extraction will be conducted independently by two reviewers in DistillerSR. A preliminary set of data items is included in table 1, which will be further specified following feedback from the disease-specific review groups. Studies' corresponding authors will be contacted if clarification is required. Disagreements will be resolved through discussion. If no consensus can be reached, a third, senior member of the research team will make the final determination.

\section{Revising data items}

If additional relevant data items are identified during the review process, they will be submitted to the team of project leads to decide whether and how to adjust the data extraction form. If included, the new data items will be extracted from all included studies.

\section{Stage 5: collating, summarising and reporting the results}

The evidence addressing each $\mathrm{RQ}$ will be mapped and analysed through narrative synthesis. Findings will be 
Table 1 Preliminary data items to extract

\begin{tabular}{|c|c|}
\hline Data items & Associated questions \\
\hline \multicolumn{2}{|c|}{ Publication details (all research questions) } \\
\hline Authors and affiliations & Who conducted the research? \\
\hline Type & In what type of literature was the study published? (Journal, grey literature, conference abstract) \\
\hline Year & When was the study published? \\
\hline Country/region & In which geographic region(s) did the study take place? \\
\hline \multicolumn{2}{|c|}{ General details (all research questions) } \\
\hline Study design & What was the study's design? \\
\hline Study aims & What were the study's aims? \\
\hline Population & $\begin{array}{l}\text { What population was studied? Were there any specific inclusion/exclusion criteria such as } \\
\text { disease severity, subtype or age? }\end{array}$ \\
\hline Study size & How many people participated in the study? \\
\hline Included DMOs & Which DMOs were measured? How and in what setting were the DMOs measured? \\
\hline \multicolumn{2}{|l|}{ Research question 1} \\
\hline Study design & $\begin{array}{l}\text { Were patients and controls matched or are the groups comparable with respect to appropriate } \\
\text { criteria (height, age, sex)? Was gait analysis controlled for gait speed? Did the study focus on a } \\
\text { specific subgroup or population? }\end{array}$ \\
\hline Differences in DMOs & $\begin{array}{l}\text { What differences in DMOs occurred (or did not occur) between the four included populations } \\
\text { and healthy controls? } \\
\text { Did these differences reach statistical significance? }\end{array}$ \\
\hline \multicolumn{2}{|l|}{ Research question 2} \\
\hline Analytical methods & $\begin{array}{l}\text { How did the authors measure the relationship between clinically relevant measures and DMOs? } \\
\text { What association measure was used? }\end{array}$ \\
\hline Clinically relevant measures & What clinically relevant measures were studied? \\
\hline Relationship strength & $\begin{array}{l}\text { What was the strength of the reported relationship between the measure and the DMO? Was the } \\
\text { association statistically significant? }\end{array}$ \\
\hline \multicolumn{2}{|l|}{ Research question 3} \\
\hline Model description & $\begin{array}{l}\text { Does the study report a multivariate analysis, a prediction model, a model based on machine- } \\
\text { learning? Which covariates were included in the model? Which analytical methods were used? }\end{array}$ \\
\hline Clinically relevant outcomes & What clinically relevant outcomes were studied to assess the DMO's prognostic value? \\
\hline Prognostic value & Did the DMO provide prognostic value with respect to the studied outcome? \\
\hline \multicolumn{2}{|l|}{ Research question 4} \\
\hline Intervention type & What intervention was studied? \\
\hline Study endpoints & $\begin{array}{l}\text { Was the DMO used as a primary, secondary or exploratory endpoint? What other primary, } \\
\text { secondary and exploratory endpoints were measured? }\end{array}$ \\
\hline Success & Was there a change in the primary endpoint between groups? \\
\hline Ability to detect change & Was the DMO able to detect a change due to the intervention (if a change occurred)? \\
\hline
\end{tabular}

DMO, digital mobility outcomes.

compiled in tables and figures where appropriate. Narrative synthesis will also be used to make comparisons between populations, disease subtypes and measurement conditions. We will also identify gaps in the evidence to inform areas of future research. Reporting will adhere to the Preferred Reporting Items for Systematic reviews and Meta-Analyses extension for Scoping Reviews ${ }^{44}$ with the exception of risk of bias and evidence strength assessments, which are not mandatory and will not be conducted in this study. ${ }^{42-44}$

\section{Stage 6: consultation}

Levac et al recommend that research teams involve stakeholders throughout review conduct, as stakeholders can provide nuanced insights beyond those reported in the literature. ${ }^{43}$ The long-term goal of Mobilise-D is to validate and qualify DMOs that can be used to assess mobility in clinical trials. While such an undertaking involves a number of diverse stakeholders, the present work could be most influenced by the perspectives of industry, patients and clinical researchers.

Patient and public involvement

Mobilise-D's pharmaceutical industry partners, patient advisory board and scientific advisory board will be consulted during review conduct and data analysis. Industry partners reviewed the RQs to ensure relevance for clinical trials and regulatory qualification. Patients were not directly involved in the design of this review. However, the Mobilise-D patient advisory board and scientific advisory board will be engaged 
during data analysis and reporting to ensure analyses align with the priorities of those groups.

\section{Discussion and limitations}

Current literature on DMOs represents a diverse set of research perspectives, resulting in a rich-though fragmented-body of literature. Therefore, we devised a broad review strategy, attempting to map the literature across clinical and technological divides. However, this strategy raises challenges of feasibility; our searches yielded tens of thousands of references. Though carefully designed, these searches may still be limited due to inconsistent terminology and reporting practices. Due to our broad strategy, we expect a high degree of heterogeneity in our results. Though challenging, this heterogeneity is also a strength, as it enables us to compare DMOs measured with various technologies under diverse walking conditions. Therefore, we predefined some of the relationships we intend to map (ie, the DMOs, measures and outcomes) while leaving other aspects of our scope open (ie, methodology, walking condition and setting). We have also applied minimal criteria such as study size, excluding the smallest studies which would crowd results. Where appropriate, we will carefully employ the reflexive strategies afforded by scoping review methodology to ensure that the items defined a-priori do not impart bias. For these reasons, this study should be interpreted as identifying clinically relevant trends within the existing literature, rather than as an exhaustive review. We will not conduct critical appraisal or metaanalysis, limiting our ability to assess the strength of existing evidence. However, we will identify topics ripe for systematic review, which should be conducted in future work.

\section{Author affiliations}

${ }^{1}$ Epidemiology, Biostatistics, and Prevention Institute, University of Zürich, Zürich, Switzerland

${ }^{2}$ Department of Neuromedicine and Movement Science, Norwegian University of Science and Technology (NTNU), Trondheim, Norway

${ }^{3}$ Barcelona Institute for Global Health (ISGlobal), Barcelona, Spain

${ }^{4}$ Universitat Pompeu Fabra (UPF), Barcelona, Spain

${ }^{5}$ Department of Neuroscience and Sheffield NIHR Translational Neuroscience BRC, Sheffield, UK

${ }^{6}$ Sheffield Teaching Hospitals NHS Foundation Trust \& University of Sheffield, Sheffield, UK

${ }^{7}$ National Heart and Lung Institute, Imperial College London, London, UK

${ }^{8}$ Department of Sport, Exercise and Rehabilitation, Faculty of Health and Life

Sciences, Northumbria University, Newcastle upon Tyne, UK

${ }^{9}$ Department of Neurology, San Raffaele Hospital, Milan, Italy

${ }^{10}$ Department of Rehabilitation Sciences, KU Leuven, Leuven, Belgium

${ }^{11}$ Department of Rehabilitation Sciences, Ghent University, Ghent, Belgium

${ }^{12}$ Department of Neurology, University Medical Center Schleswig-Holstein Campus

Kiel, Kiel, Schleswig-Holstein, Germany

${ }^{13}$ Department of Molecular Neurology, Erlangen University Hospital, Erlangen,

Bayern, Germany

${ }^{14}$ Machine Learning and Data Analytics Lab, Department of Computer Science, Friedrich-Alexander-Universität Erlangen-Nürnberg, Erlangen, Bayern, Germany

${ }^{15}$ Translational and Clinical Research Institute, Newcastle University Faculty of

Medical Sciences, Newcastle upon Tyne, UK

${ }^{16}$ Department of Clinical Gerontology, Robert Bosch Hospital, Stuttgart, BadenWürttemberg, Germany

${ }^{17}$ Department of Research \& Early Development Statistics, Bayer AG, Berlin,

Germany

${ }^{18}$ Department of Biomedical Sciences, University of Sassari, Sassari, Sardegna, Italy
${ }^{19}$ INSIGNEO Institute for in Silico Medicine, The University of Sheffield, Sheffield, UK ${ }^{20}$ Department of Mechanical Engineering, The University of Sheffield, Sheffield, UK ${ }^{21}$ Department of Rehabilitation Sciences, KU Leuven, Leuven, Flanders, Belgium

Twitter Sara Catherine Buttery @SaraButtery and Felix Kluge @kluger_felix

Acknowledgements We thank Sabine Klein, $\mathrm{PhD}$, for her invaluable input into the search strategy. We also thank those who will contribute to review conduct, who are not listed here as authors: Cameron Kirk, Gabriela Cárdenas Fuentes, Ellen Buckley, Corinna Nerz, Andrea Cereatti, Rachele Rossanigo, Sophie Breuls and Azza Ismail. A list of incoming contributors will be updated and acknowledged throughout the study process on our OSFRegistry page.

Collaborators Cameron Kirk (Translational and Clinical Research Institute, Faculty of Medical Sciences, Newcastle University, Newcastle upon Tyne, UK); Gabriela Cárdenas Fuentes (Barcelona Institute for Global Health, Barcelona, Spain); Ellen Buckley (INSIGNEO Institute for in silico Medicine, University of Sheffield, Sheffield, UK); Corinna Nerz (Department of Clinical Gerontology, Robert-Bosch-Hospital, Stuttgart, Germany); Andrea Cereatti (Department of Biomedical Sciences, University of Sassari, Italy); Rachele Rossanigo (Department of Biomedical Sciences, University of Sassari, Italy); Sophie Breuls (KU Leuven, Department of Rehabilitation Sciences, Leuven, Belgium);

Contributors AMP, AF, MAP, CB, JGA, LR and CM devised study scope and research questions. AMP, RB, MBdB, GB, SCB, NC, GdC, LDO, HD, KE, JGA, HG, CH, NH, JK, FK, SK, LL, WM, MEM-A, ASM, PP, FS, CS, LS, KS, BS, KT, TT, BV, IV, AY, CM, CB, LR, MAP and $A F$ contributed to study design. AMP wrote and edited the manuscript. AF and MAP supervised study conduct. AMP, RB, MBdB, GB, SCB, NC, GdC, LDO, HD, KE, $\mathrm{HG}, \mathrm{CH}, \mathrm{FK}, \mathrm{SK}, \mathrm{LL}, \mathrm{MEM}-\mathrm{A}, \mathrm{ASM}, \mathrm{FS}, \mathrm{LS}, \mathrm{KS}, \mathrm{KT}$ and BV will review references and extract data. All authors approved of the final manuscript.

Funding The MOBILISE-D project has received funding from the Innovative Medicines Initiative 2 Joint Undertaking under grant agreement No. 820820. This Joint Undertaking receives support from the European Union's Horizon 2020 research and innovation programme and the European Federation of Pharmaceutical Industries and Associations (EFPIA). HD is a postdoctoral research fellow of the FWO-Flanders. JGA, MBdB, LDO and SK acknowledge support from the Spanish Ministry of Science, Innovation and Universities through the 'Centro de Excelencia Severo Ochoa 2019-2023' Programme (CEX2018-000806-S), and support from the Generalitat de Catalunya through the CERCA Programme.

Disclaimer This publication reflects the authors' views and neither IMI nor the European Union, EFPIA, or any Associated Partners are responsible for any use that may be made of the information contained herein.

Competing interests AMP is a part-time employee of Merck, Sharpe and Dohme AG, but does not own stock or stock options. PP is an employee of Bayer AG, and may own stock/stock options. WM receives or received funding from the European Union, the German Federal Ministry of Education of Research, Michael J. Fox Foundation, Robert Bosch Foundation, Neuroalliance, Lundbeck and Janssen. He received speaker honoraria from Abbvie, Bayer, GlaxoSmithKline, Licher MT, Rölke Pharma and UCB, was invited to Advisory Boards of Abbvie, Biogen, Lundbeck and Market Access \& Pricing Strategy $\mathrm{GmbH}$, and is an advisory board member of the Critical Path for Parkinson's Consortium. He serves as the cochair of the MDS Technology Task Force.

Patient consent for publication Not required.

Provenance and peer review Not commissioned; externally peer reviewed.

Open access This is an open access article distributed in accordance with the Creative Commons Attribution Non Commercial (CC BY-NC 4.0) license, which permits others to distribute, remix, adapt, build upon this work non-commercially, and license their derivative works on different terms, provided the original work is properly cited, appropriate credit is given, any changes made indicated, and the use is non-commercial. See: http://creativecommons.org/licenses/by-nc/4.0/.

ORCID iDs

Ashley Marie Polhemus http://orcid.org/0000-0002-5056-5785

Sara Catherine Buttery http://orcid.org/0000-0001-9410-414X

Christian Schlenstedt http://orcid.org/0000-0002-3838-6848

\section{REFERENCES}

1 Block VAJ, Pitsch E, Tahir P, et al. Remote physical activity monitoring in neurological disease: a systematic review. PLoS One 2016;11:e0154335. 
2 Kohn CG, Baker WL, Sidovar MF, et al. Walking speed and healthrelated quality of life in multiple sclerosis. Patient 2014;7:55-61.

3 Schmid A, Duncan PW, Studenski S, et al. Improvements in speedbased gait classifications are meaningful. Stroke 2007;38:2096-100.

4 Sutliff $\mathrm{MH}$. Contribution of impaired mobility to patient burden in multiple sclerosis. Curr Med Res Opin 2010;26:109-19.

5 Motl RW, Snook EM, Wynn DR, et al. Physical activity correlates with neurological impairment and disability in multiple sclerosis. $J$ Nerv Ment Dis 2008;196:492-5.

6 Motl RW, McAuley E. Longitudinal analysis of physical activity and symptoms as predictors of change in functional limitations and disability in multiple sclerosis. Rehabil Psychol 2009;54:204-10.

7 Brodie MA, Lord SR, Coppens MJ, et al. Eight-week remote monitoring using a freely worn device reveals unstable gait patterns in older fallers. IEEE Trans Biomed Eng 2015;62:2588-94.

8 Similä $\mathrm{H}$, Immonen M, Merilahti J, et al. Gait analysis and estimation of changes in fall risk factors. Conf Proc IEEE Eng Med Biol Soc 2015;2015:6939-42.

9 Weiss A, Brozgol M, Dorfman M, et al. Does the evaluation of gait quality during daily life provide insight into fall risk? a novel approach using 3-day accelerometer recordings. Neurorehabil Neural Repair 2013;27:742-52.

10 Cesari M, Kritchevsky SB, Penninx BWHJ, et al. Prognostic value of usual gait speed in well-functioning older people--results from the Health, Aging and Body Composition Study. J Am Geriatr Soc 2005;53:1675-80.

11 Andrianopoulos V, Wouters EFM, Pinto-Plata VM, et al. Prognostic value of variables derived from the six-minute walk test in patients with COPD: results from the eclipse study. Respir Med 2015;109:1138-46.

12 Coravos A, Khozin S, Mandl KD. Developing and adopting safe and effective digital biomarkers to improve patient outcomes. NPJ Digit Med 2019;2:14.

13 Mercieca-Bebber R, King MT, Calvert MJ, et al. The importance of patient-reported outcomes in clinical trials and strategies for future optimization. Patient Relat Outcome Meas 2018;9:353-67.

14 Del Din S, Godfrey A, Mazzà C, et al. Free-living monitoring of Parkinson's disease: lessons from the field. Mov Disord 2016;31:1293-313.

15 Goldhahn J. Need for digital biomarkers in musculoskeletal trials. Digit Biomark 2017;1:82-6.

16 Stull DE, Leidy NK, Parasuraman B, et al. Optimal recall periods for patient-reported outcomes: challenges and potential solutions. Curr Med Res Opin 2009;25:929-42.

17 Eastlack ME, Arvidson J, Snyder-Mackler L, et al. Interrater reliability of videotaped observational gait-analysis assessments. Phys Ther 1991;71:465-72.

18 Wrisley DM, Walker ML, Echternach JL, et al. Reliability of the dynamic gait index in people with vestibular disorders. Arch Phys Med Rehabil 2003;84:1528-33.

19 Zhang J, Waubant E, Cutter G, et al. EDSS variability before randomization may limit treatment discovery in primary progressive MS. Mult Scler 2013;19:775-81.

20 Storm FA, Nair KPS, Clarke AJ, et al. Free-living and laboratory gait characteristics in patients with multiple sclerosis. PLoS One 2018;13:e0196463.

21 Dorsey ER, Papapetropoulos S, Xiong M, et al. The first frontier: digital biomarkers for neurodegenerative disorders. Digit Biomark 2017:1:6-13.

22 Shema-Shiratzky S, Hillel I, Mirelman A, et al. A wearable sensor identifies alterations in community ambulation in multiple sclerosis: contributors to real-world gait quality and physical activity. J Neurol : 2020. doi:10.1007/s00415-020-09759-7. [Epub ahead of print: 12 Mar 2020].

23 Del Din S, Godfrey A, Galna B, et al. Free-living gait characteristics in ageing and Parkinson's disease: impact of environment and ambulatory bout length. J Neuroeng Rehabil 2016;13:46.

24 Gold M, Amatniek J, Carrillo MC, et al. Digital technologies as biomarkers, clinical outcomes assessment, and recruitment tools in Alzheimer's disease clinical trials. Alzheimers Dement 2018;4:234-42.

25 Brognara L, Palumbo P, Grimm B, et al. Assessing gait in Parkinson's disease using wearable motion sensors: a systematic review. Diseases 2019;7. doi:10.3390/diseases7010018. [Epub ahead of print: 05 Feb 2019].

26 Gore S, Blackwood J, Guyette M, et al. Validity and reliability of Accelerometers in patients with COPD: a systematic review. $J$ Cardiopulm Rehabil Prev 2018;38:147-58.

27 Zago M, Sforza C, Bonardi DR, et al. Gait analysis in patients with chronic obstructive pulmonary disease: a systematic review. Gait Posture 2018;61:408-15.
28 Petraglia F, Scarcella L, Pedrazzi G, et al. Inertial sensors versus standard systems in gait analysis: a systematic review and metaanalysis. Eur J Phys Rehabil Med 2019;55:265-80.

29 Gordt K, Gerhardy T, Najafi B, et al. Effects of wearable Sensorbased balance and gait training on balance, gait, and functional performance in healthy and patient populations: a systematic review and meta-analysis of randomized controlled trials. Gerontology 2018;64:74-89.

30 Espay AJ, Bonato P, Nahab FB, et al. Technology in Parkinson's disease: challenges and opportunities. Mov Disord 2016;31:1272-82.

31 Maetzler W, Klucken J, Horne M. A clinical view on the development of technology-based tools in managing Parkinson's disease. Mov Disord 2016;31:1263-71.

32 Del Din S, Godfrey A, Rochester L. Validation of an accelerometer to quantify a comprehensive battery of gait characteristics in healthy older adults and Parkinson's disease: toward clinical and at home use. IEEE J Biomed Health Inform 2016;20:838-47.

33 Lipsmeier F, Taylor KI, Kilchenmann T, et al. Evaluation of smartphone-based testing to generate exploratory outcome measures in a phase 1 Parkinson's disease clinical trial. Mov Disord 2018;33:1287-97.

34 Perry B, Herrington W, Goldsack JC, et al. Use of mobile devices to measure outcomes in clinical research, 2010-2016: a systematic literature review. Digit Biomark 2018;2:11-30.

35 Pedišić Željko, Bauman A. Accelerometer-based measures in physical activity surveillance: current practices and issues. $\mathrm{Br} J$ Sports Med 2015;49:219-23.

36 Bassett DR, Troiano RP, McClain JJ, et al. Accelerometer-based physical activity: total volume per day and standardized measures. Med Sci Sports Exerc 2015;47:833-8.

37 Graham JE, Ostir GV, Fisher SR, et al. Assessing walking speed in clinical research: a systematic review. J Eval Clin Pract 2008:14:552-62.

38 Vienne-Jumeau A, Quijoux F, Vidal PP, et al. Value of gait analysis for measuring disease severity using inertial sensors in patients with multiple sclerosis: protocol for a systematic review and metaanalysis. Syst Rev 2019;8:15.

39 Lord S, Galna B, Rochester L. Moving forward on gait measurement: toward a more refined approach. Mov Disord 2013;28:1534-43.

40 Mobilise-D Consortium. Mobilise-D: connecting digital mobility assessment to clinical outcomes for regulatory and clinical endorsement, 2019. Available: http://www.mobilise-d.eu/ [Accessed 17 Sep 2019].

41 Boehme P, Hansen A, Roubenoff R, et al. How soon will digital endpoints become a cornerstone for future drug development? Drug Discov Today 2019;24:16-19.

42 Arksey H, O'Malley L. Scoping studies: towards a methodological framework. Int J Soc Res Methodol 2005;8:19-32.

43 Levac D, Colquhoun H, O'Brien KK. Scoping studies: advancing the methodology. Implement Sci 2010;5:69.

44 Tricco AC, Lillie E, Zarin W, et al. PRISMA extension for scoping reviews (PRISMA-ScR): checklist and explanation. Ann Intern Med 2018;169:467.

45 Block VJ, Lizée A, Crabtree-Hartman E, et al. Continuous daily assessment of multiple sclerosis disability using remote step count monitoring. J Neurol 2017;264:316-26.

46 Sun R, McGinnis R, Sosnoff JJ. Novel technology for mobility and balance tracking in patients with multiple sclerosis: a systematic review. Expert Rev Neurother 2018;18:887-98.

47 Middleton A, Fritz SL, Lusardi M. Walking speed: the functional vital sign. J Aging Phys Act 2015;23:314-22.

48 Fritz S, Lusardi M. White paper: "walking speed: the sixth vital sign". $J$ Geriatr Phys Ther 2009;32:2-5.

49 Studenski S, Perera S, Patel K, et al. Gait speed and survival in older adults. JAMA 2011;305:50.

50 Verghese J, Wang C, Lipton RB, et al. Quantitative gait dysfunction and risk of cognitive decline and dementia. J Neurol Neurosurg Psychiatry 2007;78:929-35.

51 Verghese J, Robbins M, Holtzer R, et al. Gait dysfunction in mild cognitive impairment syndromes. J Am Geriatr Soc 2008;56:1244-51.

52 Lord S, Galna B, Verghese J, et al. Independent domains of gait in older adults and associated motor and nonmotor attributes: validation of a factor analysis approach. $J$ Gerontol A Biol Sci Med Sci 2013;68:820-7.

53 Hollman JH, McDade EM, Petersen RC. Normative spatiotemporal gait parameters in older adults. Gait Posture 2011;34:111-8.

54 England SA, Granata KP. The influence of gait speed on local dynamic stability of walking. Gait Posture 2007;25:172-8.

55 Buzzi UH, Stergiou N, Kurz MJ, et al. Nonlinear dynamics indicates aging affects variability during gait. Clin Biomech 2003;18:435-43. 
56 Dingwell JB, Cusumano JP. Re-interpreting detrended fluctuation analyses of stride-to-stride variability in human walking. Gait Posture 2010;32:348-53.

57 Dlugonski D, Joyce RJ, Motl RW, Meanings MRW. Meanings, motivations, and strategies for engaging in physical activity among women with multiple sclerosis. Disabil Rehabil 2012;34:2148-57.

58 Fakolade A, Lamarre J, Latimer-Cheung A, et al. Understanding leisure-time physical activity: voices of people with MS who have moderate-to-severe disability and their family caregivers. Health Expect 2018;21:181-91.

59 Dobbels F, de Jong C, Drost E, et al. The proactive innovative conceptual framework on physical activity. Eur Respir $J$ 2014;44:1223-33.

60 Godinho F, Costa C, Terzian PR, et al. Forel H1 stimulation for parkinson's disease gait disorders: an instrumented gait and balance analysis. Mov Disord 2018;33:S115.

61 Bahureksa L, Najafi B, Saleh A, et al. The impact of mild cognitive impairment on gait and balance: a systematic review and metaanalysis of studies using instrumented assessment. Gerontology 2017;63:67-83.

62 McGinley JL, Baker R, Wolfe R, et al. The reliability of threedimensional kinematic gait measurements: a systematic review. Gait Posture 2009;29:360-9.
63 Fleiss JL. Measuring nominal scale agreement among many raters. Psychol Bull 1971;76:378-82.

64 Podsiadlo D, Richardson S. The timed "up \& go": a test of basic functional mobility for frail elderly persons. J Am Geriatr Soc 1991;39:142-8.

65 World Helath Organization. International classification of functioning, disability and health. Geneva, Switzerland: ICF, 2001.

66 Perry J. Ganganalyse: norm und Pathologie des Gehens. 1 edn. München: Urban \& Fischer, 2003.

67 Whittle M. Gait analysis: an introduction. 3 edn. Edinburgh: Butterworth-Heinemann, 2005.

68 Dijkstra B, Kamsma YP, Zijlstra W. Detection of gait and postures using a miniaturized triaxial accelerometer-based system: accuracy in patients with mild to moderate Parkinson's disease. Arch Phys Med Rehabil 2010;91:1272-7.

69 Iluz T, Gazit E, Herman T, et al. Automated detection of missteps during community ambulation in patients with Parkinson's disease: a new approach for quantifying fall risk in the community setting. $J$ Neuroeng Rehabil 2014;11:48.

70 Centers for Disease Control and Prevention. Principles of epidemiology: glossary, 2014. Available: https://www.cdc.gov/csels/ dsepd/ss1978/glossary.html [Accessed 26 Feb 2020]. 